\title{
Racial differences in the outcome of patients RTT with urothelial carcinoma of the upper urinary tract: an international study
}

Kazumasa Matsumoto ${ }^{1}$, Giacomo Novara ${ }^{2}$, Amit Gupta ${ }^{3}$, Vitaly Margulis ${ }^{3,4}$, Thomas J. Walton ${ }^{5}$, Marco Roscigno ${ }^{6}$, Casey $\mathrm{Ng}^{7}$, Eiji Kikuchi ${ }^{8}$, Richard Zigeuner ${ }^{9}$, Wassim Kassouf ${ }^{10}$, Hans-Martin Fritsche ${ }^{11}$, Vincenzo Ficarra ${ }^{2}$, Guido Martignoni ${ }^{12}$, Stefan Tritschler ${ }^{13}$, Joaquin Carballido Rodriguez ${ }^{14}$, Christian Seitz ${ }^{15}$, Alon Weizer ${ }^{16}$, Mesut Remzi ${ }^{17}$, Jay D. Raman ${ }^{7}$, Christian Bolenz ${ }^{18}$, Karim Bensalah ${ }^{19}$, Theresa M. Koppie ${ }^{20}$, Pierre I. Karakiewicz ${ }^{21}$, Christopher G. Wood ${ }^{4}$, Francesco Montorsi ${ }^{6}$, Masatsugu Iwamura ${ }^{1}$ and Shahrokh F. Shariat ${ }^{22}$ ${ }^{1}$ Kitasato University School of Medicine, Sagamihara, Kanagawa, Japan; ${ }^{2}$ University of Padua, Padua, Italy; ${ }^{3}$ University of Texas, Southwestern Medical Center, Dallas, TX, ${ }^{4}$ University of Texas MD Anderson Cancer Center, Houston, TX, USA; ${ }^{5}$ Royal Derby Hospital, Derby, UK; ${ }^{6}$ Vita-Salute University, Milan, Italy; ${ }^{7}$ Division of Urology, Penn State Milton S. Hershey Medical Center, Hershey, PA, USA; ${ }^{8}$ Keio University School of Medicine, Tokyo, Japan; ${ }^{9}$ Medical University Graz, Graz, Austria; ${ }^{10}$ McGill University Health Centre, Montréal, Quebec, Canada; ${ }^{11}$ Caritas St. Josef Medical Center, University of Regensburg, Regensburg, Germany; ${ }^{12}$ University of Verona, Verona, Italy; ${ }^{13}$ LudwigMaximilians-University, Klinikum Grosshadern, Munich, Germany; ${ }^{14}$ Hospital Universitario Puerta de HierroMajadahonda, Universidad Autónoma de Madrid, Madrid, Spain; ${ }^{15}$ General Hospital Bolzano, Bolzano, Italy; ${ }^{16}$ University of Michigan, Ann Arbor, MI, USA; ${ }^{17}$ Medical University of Vienna, Vienna, Austria; ${ }^{18}$ Mannheim Medical Center, University of Heidelberg, Germany; ${ }^{19}$ University of Rennes, Rennes, France; ${ }^{20}$ University of California Davis, Sacramento, CA, USA; ${ }^{21}$ University of Montréal, Montréal, Quebec, Canada; ${ }^{22}$ Weill Medical College of Cornell University, New York, NY, USA

Accepted for publication 26 November 2010

K.M. and G.N. equally contributed to the study.

Study Type - Therapy (case series) Level of Evidence 4

\section{OBJECTIVE}

- To assess the impact of differences in ethnicity on clinico-pathological characteristics and outcomes of patients with upper urinary tract urothelial carcinoma (UTUC) in a large multi-center series of patients treated with radical nephroureterectomy (RNU).

\section{MATERIALS AND METHODS}

- We retrospectively collected the data of 2163 patients treated with RNU at 20 academic centres in America, Asia, and Europe.

- Univariable and multivariable Cox regression models addressed recurrence-free

What's known on the subject? and What does the study add? The differential effect of ethnicity on clinico-pathological features and cancer-related outcomes remains uninvestigated in upper tract urothelial carcinoma.

Ethnicity was not an independent predictor of either recurrence or cancer-related death in upper tract urothelial carcinoma.

survival (RFS) and cancer-specific survival (CSS).

\section{RESULTS}

- In all, 1794 (83\%) patients were Caucasian and 369 (17\%) were Japanese. All the main clinical and pathological features were significantly different between the two ethnicities.

- The median follow-up of the whole cohort was 36 months. At last follow-up, 554 patients (26\%) developed disease recurrence and 461 (21\%) were dead from UTUC.

- The 5-year RFS and CSS estimates were $71.5 \%$ and $74.2 \%$, respectively, for Caucasian patients compared with $68.8 \%$ and $75.4 \%$, respectively, for Japanese patients.

- On univariable Cox regression analyses, ethnicity was not significantly associated with either RFS $(P=0.231)$ or CSS $(P=0.752)$. 
ETHNIC DIFFERENCES IN UPPER TRACT UROTHELIAL CARCINOMA OUTCOMES)

\footnotetext{
- On multivariable Cox regression analyses that adjusted for the effects of age, gender, surgical type, T stage, grade, tumour architecture, presence of concomitant carcinoma in situ, lymphovascular invasion, tumour necrosis, and lymph node status, ethnicity was not associated with either RFS (hazard ratio $[\mathrm{HR}] 1.1 ; P=0.447)$ or CSS (HR 1.0; $P=0.908)$.
}

\section{CONCLUSIONS}

- There were major differences in the clinico-pathological characteristics of Caucasian and Japanese patients.

- However, RFS and CSS probabilities were not affected by ethnicity and race was not an independent predictor of either recurrence or cancer-related death.

\section{KEYWORDS}

race, prognosis, urinary tract cancer, urothelial carcinoma, nephroureterectomy, recurrence

\section{INTRODUCTION}

The incidence of cancer and its biological behaviour varies considerably among ethnicities and between geographic areas [1-3]. For example, epidemiological studies originating from the USA show that tumour stage and mortality rates for many cancers including those arising from breast, colon and rectum, kidney, prostate, and lung are highest in African-Americans followed by Whites and are lowest in Asians/Pacific Islanders [4-6]. Similarly, for bladder urothelial carcinoma (UC), African-Americans have worse overall survival when compared with Caucasian patients [7-10]. Although much of this difference has been attributed to higher stage at presentation in African-Americans $[8,9]$, outcome disparities still persist after controlling for tumour stage, grade, treatment effect, and other cause mortality [10]. Potential differences contributing to these disparities among ethnicities and geographic locations include delays in presentation/diagnosis, inherent biological behaviour of the tumour, socioeconomic status, lack of adequate health care access, differences in exposure to environmental risk factors such as smoking, and social support. For upper urinary tract UC (UTUC), to our knowledge, the differential effect of ethnicity on clinico-pathological features and cancer-related outcomes remains uninvestigated.

Therefore, the purpose of the present study was to assess the effect of differences in ethnicity on clinico-pathological characteristics and outcomes of patients with UTUC in a large multi-center series of patients treated with radical nephroureterectomy (RNU) in three continents (America, Asia, and Europe), encompassing nine countries (USA, Canada, Japan, Austria, France, England, Germany, Italy, and Spain).

\section{PATIENTS AND METHODS}

This was an Institutional Review Boardapproved study with all participating sites providing the necessary institutional data sharing agreements before initiation of the study. In all, 20 academic centres worldwide provided data. A computerized databank was generated for data transfer. After combining the datasets, reports were generated for each variable to identify data inconsistencies and other data integrity problems. Through regular communication with all sites, resolution of all identified anomalies was achieved before analysis. Before final analysis, the database was frozen, and the final data set was produced for the present analysis.

The database comprised 2217 patients who underwent RNU with ipsilateral bladder cuff resection between 1987 and 2008. After exclusion of patients in whom ethnicity was unknown (six patients), and of the few patients that were African-American (27) or Hispanic (21), the 2163 patients left were the subjects of the present analysis.

Surgery was performed by several surgeons according to the standard criteria for RNU, i.e. extrafascial dissection of the kidney with the entire length of ureter and adjacent segment of the bladder cuff. The hilar and regional LNs adjacent to the ipsilateral great vessel generally were resected together with enlarged LNs if abnormal on preoperative CT or palpable intraoperatively. Extended lymphadenectomy was not routinely performed.

All surgical specimens were processed according to standard pathological procedures at each institution. Tumours were staged according to the American Joint Committee on Cancer-Union Internationale Contre le Cancer TNM classification. [11]. Tumour grading was assessed according to the to the $1998 \mathrm{WHO} /$ International Society of
Urologic Pathology consensus classification [12].

Patients were generally followed-up every 34 months for the first year after RNU, every 6 months from the second through to the fifth year, and annually thereafter. Follow-up consisted of a history, physical examination, routine blood work and serum chemistry studies, urinary cytology, chest radiography, cystoscopic evaluation of the urinary bladder, and radiographic evaluation of the contralateral upper urinary tract. Elective bone scan, chest $C T$, and MRI were performed when clinically indicated.

Disease recurrence was defined as local failure in the operative site, regional LNs, or distant metastasis. Bladder recurrences were not considered in the analysis of the recurrence-free survival (RFS) rate. Cause of death was determined by the treating physicians, by chart review corroborated by death certificates, or by death certificates alone. Most patients who were identified as having died from UTUC had progressive, widely disseminated metastases at the time of death. Patients who died in the perioperative period (i.e. death within 30 days of surgery) were censored at time of death for cancerspecific survival (CSS) analyses.

The Fisher's exact test and the chi-square test were used to evaluate the association between categorical variables. Differences in variables with a continuous distribution across dichotomous categories were assessed using the Mann-Whitney U-test. The KaplanMeier method was used to calculate survival functions, and differences were assessed with the log-rank statistic. Univariable and multivariable Cox regression models addressed time to recurrence and cancerspecific mortality after RNU. Statistical significance in this study was set as $P \leq 0.05$. All reported $P$ values are two-sided. 


\section{RESULTS}

\section{ASSOCIATION OF ETHNICITY WITH CLINICAL AND PATHOLOGICAL FEATURES}

In all, 1794 (83\%) patients were Caucasian and 369 (17\%) were Japanese. Table 1 shows the association of clinical and pathological features with ethnicity. Patients' age, gender, tumour stage, tumour grade, prevalence of lymphovascular invasion, tumour architecture, prevalence of tumour necrosis, presence of concomitant carcinoma in situ (CIS), and use of adjuvant chemotherapy were significantly different between the two ethnicities (all $P<0.05$ ). Moreover, also follow-up duration from RNU was slightly longer in Japanese patients $(P=0.039)$.

\section{ASSOCIATION OF RACE WITH CLINICAL OUTCOMES}

The median (interquartile range, IQR) followup of the whole cohort was 36 (15.3-71.1) months. At last follow-up, 554 patients (26\%) had developed disease recurrence and 461 (21\%) were dead from UTUC. Moreover, 301 patients (14\%) had noncancer-related deaths. The median (IQR) follow-up for patients alive at last follow-up was 45 (20-81) months. The overall 5- and 10-year RFS estimates were $71 \%$ (standard error, SE 1.1\%) and 67\% (SE $1.3 \%$ ) respectively. The overall 5 - and 10 -year CSS estimates were 74.4\% (SE 1.1\%) and $69.1 \%$ (SE 1.4\%), respectively.

The 5-year RFS and CSS rates were 71.5\% (SE 1.2\%) and 74.2\% (SE 1.2\%), respectively, for Caucasian patients compared with 68.8\% (SE 2.7\%) and 75.4\% (SE 2.6\%), respectively, for Japanese patients (Fig. 1A, $B$, respectively; $P$ value 0.23 for RFS and 0.75 for CSS).

Tables 2 and 3 summarize univariable and multivariable analyses for prediction of RFS and CSS, respectively. On univariable Cox regression analyses, ethnicity was not significantly associated with either RFS ( $P=$ $0.231)$ or CSS $(P=0.752)$. On multivariable Cox regression analyses that adjusted for the effects of age, gender, surgical type, T stage, grade, tumour architecture, presence of concomitant CIS, lymphovascular invasion, tumour necrosis, and lymph node (LN) status, ethnicity was not associated with either RFS (hazard ratio $[H R] 1.1 ; P=0.447)$ or CSS (HR 1.0; $P=0.908)$.

\begin{tabular}{|c|c|c|c|c|}
\hline \multirow[b]{2}{*}{ Variable } & \multirow[b]{2}{*}{ Cases } & \multicolumn{2}{|l|}{ Race } & \multirow[b]{2}{*}{$P$} \\
\hline & & Caucasian & Japanese & \\
\hline Number of patients (\%) & $2163(100)$ & $1794(83)$ & $369(17)$ & \\
\hline Median (IQR) age, years & $69(61-76)$ & $69.9(62-76)$ & $66.3(58.3-74)$ & $<0.001$ \\
\hline Gender, $n(\%)$ & & & & $<0.001$ \\
\hline - Male & $1478(68)$ & $1186(66)$ & 292 (79) & \\
\hline - Female & $685(32)$ & $608(34)$ & 77 (21) & \\
\hline Type of RNU, $n(\%)$ & & & & 0.821 \\
\hline - Open & $1790(83)$ & $1486(83)$ & $304(82)$ & \\
\hline - Laparoscopic & $373(17)$ & $308(17)$ & 65 (18) & \\
\hline LN dissection, $n(\%)$ & & & & 0.162 \\
\hline - Performed & $604(28)$ & $490(27)$ & $114(31)$ & \\
\hline - Not performed & $1559(72)$ & 1304 (73) & $255(69)$ & \\
\hline Pathological stage, $n(\%)$ & & & & 0.003 \\
\hline - pT0 & $10(0.5)$ & $10(0.5)$ & 0 & \\
\hline - pTa & $450(21)$ & $382(21)$ & $68(18)$ & \\
\hline - pTis & $36(2)$ & $26(1.5)$ & $10(3)$ & \\
\hline - pT1 & $488(23)$ & $426(24)$ & $62(17)$ & \\
\hline - pT2 & $401(18.5)$ & 330 (18) & $71(19)$ & \\
\hline - pT3 & $667(31)$ & $535(30)$ & $132(36)$ & \\
\hline - pT4 & $111(5)$ & $85(5)$ & $26(7)$ & \\
\hline Grade, $n(\%)$ & & & & $<0.001$ \\
\hline - Low & $655(30)$ & 587 (33) & $68(18)$ & \\
\hline - High & $1508(70)$ & $1207(67)$ & $301(82)$ & \\
\hline Lymphovascular invasion, $n(\%)$ & & & & 0.001 \\
\hline - Absent & $1682(78)$ & $1372(77)$ & $310(84)$ & \\
\hline - Present & $481(22)$ & $422(23)$ & $59(16)$ & \\
\hline Tumour architecture, $n(\%)$ & & & & $<0.001$ \\
\hline - Papillary & $1660(77)$ & $1351(75)$ & $309(84)$ & \\
\hline - Sessile & $503(23)$ & $443(25)$ & $60(16)$ & \\
\hline Tumour necrosis, $n(\%)$ & & & & $<0.001$ \\
\hline - Absent & $1667(77)$ & $1354(76)$ & $313(85)$ & \\
\hline - Present & $496(23)$ & $440(24)$ & $56(16)$ & \\
\hline Concomitant $\mathrm{CIS}, n(\%)$ & & & & 0.014 \\
\hline - Absent & $1671(77)$ & $1368(76)$ & $303(82)$ & \\
\hline - Present & $492(23)$ & $426(24)$ & $66(18)$ & \\
\hline Median (IQR) no. removed LNs & $4(2-8)$ & $4(2-8)$ & $3(2-7)$ & 0.147 \\
\hline LN stage, $n(\%)$ & & & & 0.247 \\
\hline- NO & 419 (19) & $336(19)$ & $83(23)$ & \\
\hline$-N x$ & $1559(72)$ & $1304(73)$ & $255(69)$ & \\
\hline$-\mathrm{N}+$ & $185(9)$ & $154(8)$ & $31(8)$ & \\
\hline Adjuvant chemotherapy*, $n(\%)$ & & & & $<0.001$ \\
\hline - no & 1928 (89) & $1629(91)$ & $299(81)$ & \\
\hline - yes & $224(10)$ & $154(9)$ & 70 (19) & \\
\hline Median (IQR) follow-up, months & $36(15.3-71.1)$ & 35.1 (15.5-69) & $38(15-84)$ & 0.039 \\
\hline
\end{tabular}

Re-analysis of the data after exclusion of 224 patients who received adjuvant chemotherapy yielded consistent statistical patterns of H.R. and $P$ values for ethnicity (HR $1.1 ; P=0.685$ for RFS; H.R. $0.9 ; P=0.730$ for CSS). Further subgroup analyses in patients with pathologically localized, locallyadvanced, No/Nx, or N positive disease yielded consistent statistical patterns (data not shown).

\section{DISCUSSION}

In the present multi-institutional study involving about 2200 patients with UTUC 
FIG. 1. A

Kaplan-Meier curves of RFS and

CSS stratified by patients' race in

2163 patients treated with RNU

and ipsilateral bladder-cuff excision for UTUC. Green,

Caucasian patients; blue,

Japanese patients. Log-rank $P$ values 0.23 for RFS and 0.75 for

CSS.

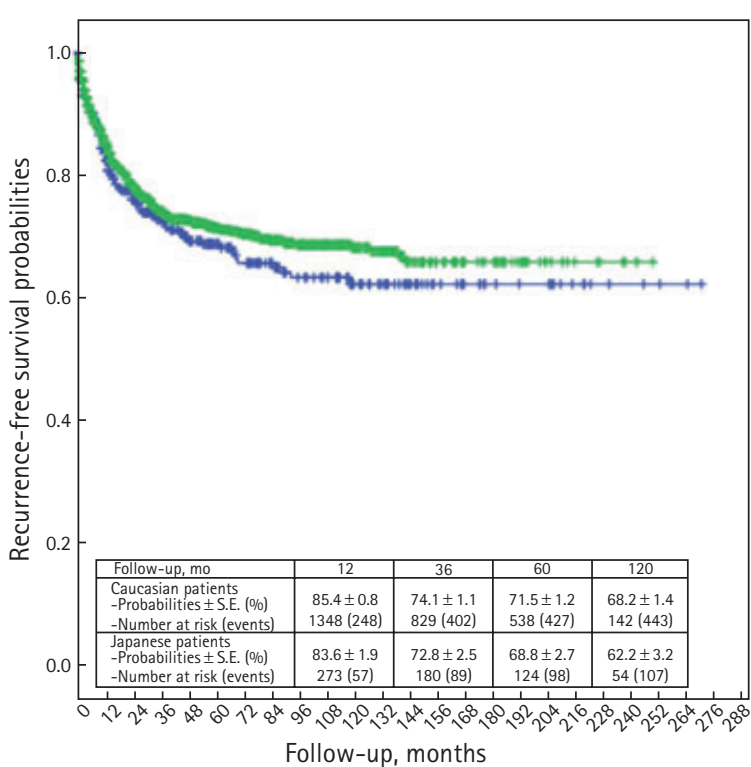

B

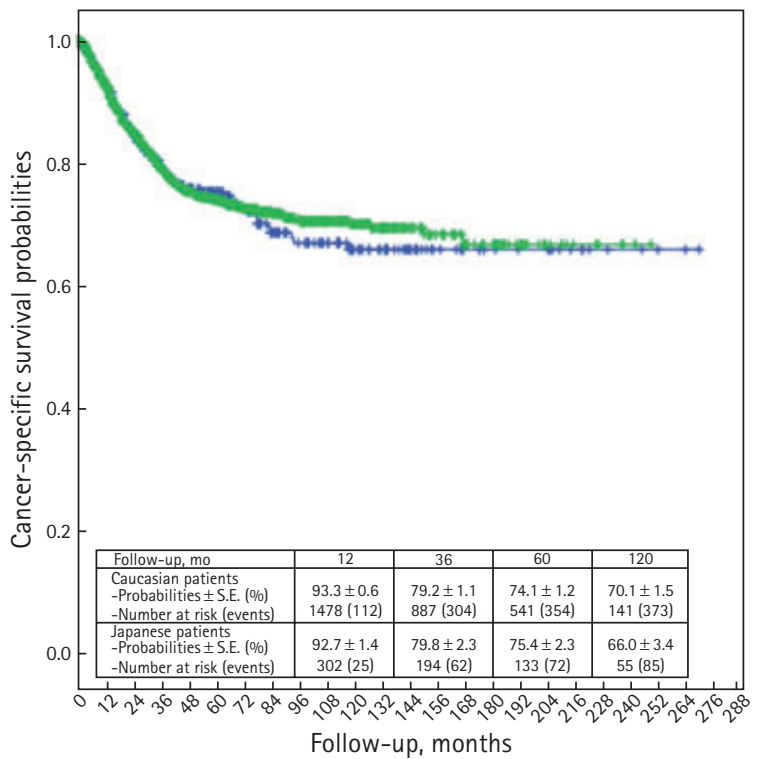

undergoing RNU there were major differences in the clinico-pathological characteristics of Caucasian and Japanese patients. However, RFS and CSS probabilities were not affected by ethnicity and race was not an independent predictor of either recurrence or cancerrelated death, once adjusted for the effects of other clinical and pathological covariates.

It is well established by epidemiological studies that rates of cancers incidence and deaths vary considerably among racial groups. Data from the National Center for
Health Statistics concerning statistics collected from 1930 to 2006 in the USA showed that African-Americans had incidence and death rates that were consistently higher than in Whites for all cancer sties, excluding breast, lung and kidney cancers $[4,5]$. Specifically, all site incidence and mortality rates were 651.5 and 313 per 100000 in male African-Americans and 389 and 186.7 per 100000 in female AfricanAmericans, respectively, compared with 551.4 and 230.7 per 100000 in male White and with 423.6 and 159.2 per 100000 in female White, respectively. Significantly lower figures, indeed, were shown for Asian-American and Hispanic, compared with both AfricanAmerican and White [4].

However, specific epidemiological data for UTUC are missing, due to the low prevalence of the disease and, above all, to the lack of a specific code able to distinguish RCC and renal pelvis UTUC in the International Classification of Diseases. However, figures for bladder UC reconfirmed a similar trend, with significantly higher incidence and mortality rates in White patients (22.8 and 4.5 per 100000 , respectively), compared with African-American (12.8 and 3.7 per 100000 , respectively), Asian-American (9.3 and 1.7 per 100000 , respectively), and Hispanic patients (11.4 and 2.4 per 100 000, respectively) [6]. In the present analysis, there was a high prevalence of non-organ confined (43\%) and high-grade (82\%) disease in Asiatic patients, which is in agreement with previous report from Taiwan [13,14], where pT3-4 disease might be as frequent as 50\%. Several hypotheses can be made to explain the differences in the clinical and pathological characteristics of UTUC at presentation. For example, environmental and occupational exposures, lifestyle choices such as smoking and nutrition, and socioeconomic and cultural factors involving access to and the willingness to seek care may account for such differences [15]. However, despite the large number of patients, we were unable to find any statistically significant differences in RFS and CSS probabilities, between these two racial groups. Moreover, the few AfricanAmerican and Hispanic patients in our dataset prevented us from including them in the analysis.

For the other predictors of RFS and CSS, in our multivariable models we showed that patients' age at surgery, pathological stage of the primary tumour, tumour grade, presence of lymphovascular invasion, tumour architecture, and LN stage were independent predictors of both recurrence and survival, while type of surgery was significantly associated only with cancer-related death. The data of the present study reproduced and validated those previously available on $\mathrm{pT}$ stage and grade [16,17], Iymphovascular invasion [18,19], architecture [20], and LN stage [21]. Notably, patients undergoing laparoscopic RNU had worse CSS once adjusted for the effects of the other covariates. The data echoes the findings of 
Simone et al. [22], who showed that laparoscopic surgery may jeopardize the outcome of patients with locally advanced disease. However, similar data were not reproduced in large multi-institutional comparative studies $[23,24]$.

There are several limitations to the present study. First and foremost are the limitations inherent to retrospective analyses. Although we have done multiple internal and external reviews of our consortium dataset, we excluded from this analysis patients for whom we could not obtain complete information, which could possibly create selection bias. In addition, the population in this study underwent RNU by multiple surgeons, indication and extension of LN dissection were not standardized, and the follow-up lacked uniformity. However, all surgeons operated at selected centres with significant experience in UC management, which might increase the external validity of the data, compared with the single-centre, singlesurgeon setting. Similarly, whereas it may be preferable for a single pathologist specialized in genitourinary pathology to review each specimen, the multi-centric nature of the present study and the use of institutional pathological interpretation possibly make the results more relevant in the daily clinical setting. Nevertheless, all specimens were examined by dedicated genitourinary pathologists at selected centres. Another limitation is that there were few Hispanic and African-American patients, which prevented us from drawing conclusions on these racial groups. In addition we did not control for important factors such as patient comorbidity, socioeconomic status, marital status and other measures of social support, body mass index, and smoking status, which have been shown to be of interest in some reports [25-27].

In conclusion, there were major differences in the clinico-pathological characteristics of Caucasian and Japanese patients. However, RFS and CSS probabilities were not affected by ethnicity and race was not an independent predictor of either recurrence or cancerrelated death, once adjusted for the effects of other clinical and pathological covariates. In terms of racial differences of biological behaviour, future research is warranted to take into account the components, including environmental and occupational exposures, lifestyle choices, socioeconomic, cultural and genetic factors.
TABLE 2 Univariable and multivariable Cox regression analyses of race for prediction of disease recurrence in 2163 patients treated with RNU and ipsilateral bladder-cuff excision for UTUC (554 recurrences)

\begin{tabular}{|c|c|c|c|c|}
\hline \multirow[b]{2}{*}{ Variable } & \multicolumn{2}{|c|}{ Univariable analysis } & \multicolumn{2}{|c|}{ Multivariable analysis } \\
\hline & $\overline{\mathrm{HR}}(95 \% \mathrm{Cl})$ & $P$ & $\overline{\mathrm{HR}}(95 \% \mathrm{Cl})$ & $P$ \\
\hline$\overline{\text { Age }}$ & $1.01(1-1.02)$ & 0.001 & $1.01(1.01-1.02)$ & 0.017 \\
\hline Race & $1.1(0.9-1.4)$ & 0.231 & $1.1(0.9-1.4)$ & 0.447 \\
\hline Gender & $1.1(0.9-1.4)$ & 0.139 & $1.1(0.9-1.3)$ & 0.558 \\
\hline Type of RNU (open vs lap.) & $0.8(0.6-1.0)$ & 0.048 & $0.9(0.7-1.2)$ & 0.437 \\
\hline Pathological stage: & & $<0.001$ & & $<0.001$ \\
\hline - pTa/Tis & 1, Reference & - & 1, Reference & - \\
\hline - pT1 & $1.6(1.01-2.5)$ & 0.043 & $1.3(0.8-2.0)$ & 0.309 \\
\hline - pT2 & $4.2(2.9-6.3)$ & $<0.001$ & $3.0(1.9-4.4)$ & $<0.001$ \\
\hline - pT3 & $9.2(6.4-13.2)$ & $<0.001$ & $5.1(3.4-7.7)$ & $<0.001$ \\
\hline - pT4 & $37.6(24.9-56.7)$ & $<0.001$ & $14.7(9.2-23.5)$ & $<0.001$ \\
\hline Grade & $4.1(3.2-5.3)$ & $<0.001$ & $1.8(1.4-2.4)$ & $<0.001$ \\
\hline Lymphovascular invasion & $3.6(3.0-4.2)$ & $<0.001$ & $1.4(1.2-1.8)$ & $<0.001$ \\
\hline Tumour architecture & $3.8(3.2-4.5)$ & $<0.001$ & $1.4(1.1-1.7)$ & 0.003 \\
\hline Tumour necrosis & $2.2(1.9-2.6)$ & $<0.001$ & $0.9(0.7-1.1)$ & 0.205 \\
\hline Concomitant $\mathrm{CIS}$ & $1.6(1.4-2.0)$ & $<0.001$ & $1.1(0.9-1.4)$ & 0.178 \\
\hline LN stage: & & $<0.001$ & & $<0.001$ \\
\hline - NO & 1, Reference & - & 1, Reference & - \\
\hline$-N x$ & $0.9(0.8-1.2)$ & 0.954 & $1.3(0.9-1.6)$ & 0.054 \\
\hline$-\mathrm{N}+$ & $4.6(3.5-6.0)$ & $<0.001$ & $2.1(1.6-2.8$ & $<0.001$ \\
\hline
\end{tabular}

TABLE 3 Univariable and multivariable Cox regression analyses of patients' race for prediction of cancerspecific mortality in 2163 patients treated with RNU and ipsilateral bladder-cuff excision for UTUC (461 cancer-specific deaths)

\begin{tabular}{|c|c|c|c|c|}
\hline \multirow[b]{2}{*}{ Variable } & \multicolumn{2}{|c|}{ Univariable analysis } & \multicolumn{2}{|c|}{ Multivariable analysis } \\
\hline & $\overline{\mathrm{HR}}(95 \% \mathrm{Cl})$ & $P$ & $\mathrm{HR}(95 \% \mathrm{Cl})$ & $P$ \\
\hline$\overline{\text { Age }}$ & $1.02(1.01-1.03)$ & $<0.001$ & $1.02(1.01-1.03)$ & $<0.001$ \\
\hline Race & $1.1(0.8-1.3)$ & 0.752 & $1.01(0.8-1.3)$ & 0.908 \\
\hline Gender & $1.1(0.9-1.3)$ & 0.542 & $0.9(0.7-1.1)$ & 0.407 \\
\hline Type of RNU (open vs lap.) & $0.6(0.4-0.9)$ & 0.004 & $0.6(0.5-0.9)$ & 0.012 \\
\hline Pathological stage: & & $<0.001$ & & $<0.001$ \\
\hline - pTa/Tis & 1, Reference & - & 1, Reference & - \\
\hline - pT1 & $1.5(0.9-2.4)$ & 0.145 & $1.2(0.7-1.9)$ & 0.570 \\
\hline - pT2 & $4.5(2.9-7.1)$ & $<0.001$ & $3.2(2.0-5.1)$ & $<0.001$ \\
\hline - pT3 & $10.1(6.7-15.2)$ & $<0.001$ & $5.9(3.8-9.2)$ & $<0.001$ \\
\hline - pT4 & $43.3(27.4-68.4)$ & $<0.001$ & $17.4(10.3-29.4)$ & $<0.001$ \\
\hline Grade & $4.0(3.0-5.2)$ & $<0.001$ & $1.8(1.3-2.4)$ & $<0.001$ \\
\hline Lymphovascular invasion & $3.9(3.3-4.7)$ & $<0.001$ & $1.6(1.3-2.0)$ & 0.001 \\
\hline Tumour architecture & $4.0(3.3-4.8)$ & $<0.001$ & $1.3(1.02-1.6)$ & 0.029 \\
\hline Tumour necrosis & $2.4(1.9-2.9)$ & $<0.001$ & $0.9(0.7-1.1)$ & 0.380 \\
\hline Concomitant CIS & $1.5(1.2-1.8)$ & $<0.001$ & $1.0(0.8-1.3)$ & 0.766 \\
\hline LN stage & & $<0.001$ & & $<0.001$ \\
\hline - No & 1, Reference & - & 1, Reference & - \\
\hline$-N x$ & $1.1(0.8-1.4)$ & 0.680 & $1.4(1.1-1.9)$ & 0.008 \\
\hline$-\mathrm{N}+$ & $4.9(3.6-6.6)$ & $<0.001$ & $2.1(1.5-2.9)$ & $<0.001$ \\
\hline
\end{tabular}

Lap, laparoscopic. 


\section{CONFLICT OF INTEREST}

None declared.

\section{REFERENCES}

1 Aben KK, Kiemeney LA. Epidemiology of bladder cancer. Eur Urol 1999; 36: 66072

2 Konety BR, Joyce GF, Wise M. Bladder and Upper Tract Urothelial Cancer. In Litwin MA, Saigal CS eds. Urologic Diseases in America 2007, US Department of Health and Human Services, Public Health Service, National Institutes of Health, National Institutes of Diabetes and Digestive and Kidney Diseases. Washington, DC: US Government Printing Office, 2007: 225-79, NIH Publication No. 07-5512

3 Madeb R, Messing EM. Gender, racial and age differences in bladder cancer incidence and mortality. Urol Onco/ 2004; 22: 86-92

4 Jemal A, Siegel R, Ward E, Hao Y, Xu J, Thun MJ. Cancer statistics, 2009. CA Cancer J Clin 2009; 59: 225-49

5 Coleman MP, Quaresma M, Berrino F et al. Cancer survival in five continents: a worldwide population-based study (CONCORD). Lancet Oncol 2008; 9: 73056

6 Surveillance Epidemiology and End Results. Incidence and Mortality by Race/ Ethnicity. Available at: http://seer.cancer. gov/csr/1975_2006/results_merged/ topic_race_ethnicity.pdf. Accessed January 2011

7 Lee CT, Dunn RL, Williams C, Underwood W III. Racial disparity in bladder cancer: trends in tumor presentation at diagnosis. J Urol 2006; 176: 927-34

8 Prout GR Jr, Wesley MN, Greenberg RS et al. Bladder cancer: race differences in extent of disease at diagnosis. Cancer 2000; 89: 1349-58

9 Prout GR Jr, Wesley MN, McCarron PG et al. Survival experience of black patients and white patients with bladder carcinoma. Cancer 2004; 100: 621-30

10 Underwood W III, Dunn RL, Williams C, Lee CT. Gender and geographic influence on the racial disparity in bladder cancer mortality in the US. J Am Coll Surg 2006; 202: 284-90

11 Greene FL, Page DL, Fleming ID eds. American Joint Committee on Cancer (AJCC) Staging Manual, 6th edn. Philadelphia: Springer, 2002

12 Epstein Jl, Amin MB, Reuter VR, Mostofi FK. The World Health Organization/ International Society of Urological Pathology consensus classification of urothelial (transitional cell) neoplasms of the urinary bladder: Bladder Consensus Conference Committee. Am J Surg Pathol 1998; 22: 1435-48

13 Kang $\mathrm{CH}, \mathrm{Yu}$ TJ, Hsieh $\mathrm{HH}$ et al. The development of bladder tumors and contralateral upper urinary tract tumors after primary transitional cell carcinoma of the upper urinary tract. Cancer 2003; 98: 1620-6

14 Li CC, Chang TH, Wu WJ et al. Significant predictive factors for prognosis of primary upper urinary tract cancer after radical nephroureterectomy in Taiwanese patients. Eur Uro/ 2008; 54 : 1127-34

15 Colin P, Koenig P, Ouzzane A et al. Environmental factors involved in carcinogenesis of urothelial cell carcinomas of the upper urinary tract. BJU Int 2009; 104: 1436-40

16 Zigeuner R, Pummer K. Urothelial carcinoma of the upper urinary tract: surgical approach and prognostic factors. Eur Uro/ 2008; 53: 720-31

17 Margulis V, Shariat SF, Matin SF et al. Outcomes of radical nephroureterectomy: a series from the Upper Tract Urothelial Carcinoma Collaboration. Cancer 2009; 115: 1224-33

18 Kikuchi E, Margulis V, Karakiewicz PI et al. Lymphovascular invasion predicts clinical outcomes in patients with nodenegative upper tract urothelial carcinoma. J Clin Oncol 2009; 27: 612-8

19 Novara G, Matsumoto K, Kassouf W et al. Prognostic role of lymphovascular invasion in patients with urothelial carcinoma of the upper urinary tract: an international validation study. Eur Urol 2010; 57: 1064-71

20 Remzi M, Haitel A, Margulis V et al. Tumour architecture is an independent predictor of outcomes after nephroureterectomy: a multi- institutional analysis of 1363 patients. BJU Int 2008; 103: 307-11

21 Roscigno M, Shariat SF, Margulis V et al. The extent of lymphadenectomy seems to be associated with better survival in patients with nonmetastatic upper-tract urothelial carcinoma: how many lymph nodes should be removed? Eur Urol 2009; 56: 512-8

22 Simone G, Papalia R, Guaglianone S et al. Laparoscopic versus open nephroureterectomy: perioperative and oncologic outcomes from a randomised prospective study. Eur Urol 2009; 56: 520-6

23 Capitanio U, Shariat SF, Isbarn H et al. Comparison of oncologic outcomes for open and laparoscopic nephroureterectomy: a multiinstitutional analysis of 1249 cases. Eur Urol 2009; 56: 1-9

24 Walton TJ, Novara G, Matsumoto K et al. Oncological outcomes after laparoscopic and open radical nephroureterectomy: results from an international cohort. BJU Int 2010; in press [Epub ahead of print]; doi: 10.1111/ j.1464-410X.2010.09826.x

25 Gore JL, Kwan L, Saigal CS, Litwin MS. Marriage and mortality in bladder carcinoma. Cancer 2005; 104: 1188 94

26 Behavioral Risk Factor Surveillance System (BRFSS), United States. 2007. Current Smokers. 2007.

27 Carmona R, Gfroerer J, Caraballo R et al. Prevalence of cigarette use among 14 racial/ethnic populations - United States, 1999-2001. Morb Mortal Wkly Rep 2004; 53: $49-52$

Correspondence: Shahrokh F. Shariat, Departments of Urology and Medical Oncology, Weill Cornell Medical Center, NewYork-Presbyterian, New York, NY, USA. e-mail: sfshatiat@gmail.com or sfs2002@med.cornell.edu

Abbreviations: (UT)UC, (upper urinary tract) urothelial carcinoma; RNU, radical nephroureterectomy; RFS, recurrence-free survival; CSS, cancer-specific survival; CIS, carcinoma in situ; $I Q R$, interquartile range; $S E$, standard error; LN, lymph node; HR, hazard ratio. 